\title{
Residual metabolic tumor activity after chemo- radiotherapy is mainly located in initially high FDG uptake areas in rectal cancer
}

Citation for published version (APA):

van den Bogaard, J., Janssen, M. H. M., Janssens, G. O., Buijsen, J., Reniers, B., Lambin, P., Lammering, G., \& Ollers, M. C. (2011). Residual metabolic tumor activity after chemo-radiotherapy is mainly located in initially high FDG uptake areas in rectal cancer. Radiotherapy and Oncology, 99(2), 137141. https://doi.org/10.1016/j.radonc.2011.04.004

Document status and date:

Published: 01/05/2011

DOI:

10.1016/j.radonc.2011.04.004

Document Version:

Publisher's PDF, also known as Version of record

\section{Document license:}

Taverne

Please check the document version of this publication:

- A submitted manuscript is the version of the article upon submission and before peer-review. There can be important differences between the submitted version and the official published version of record.

People interested in the research are advised to contact the author for the final version of the publication, or visit the DOI to the publisher's website.

- The final author version and the galley proof are versions of the publication after peer review.

- The final published version features the final layout of the paper including the volume, issue and page numbers.

Link to publication

\footnotetext{
General rights rights.

- You may freely distribute the URL identifying the publication in the public portal. please follow below link for the End User Agreement:

www.umlib.nl/taverne-license

Take down policy

If you believe that this document breaches copyright please contact us at:

repository@maastrichtuniversity.nl

providing details and we will investigate your claim.
}

Copyright and moral rights for the publications made accessible in the public portal are retained by the authors and/or other copyright owners and it is a condition of accessing publications that users recognise and abide by the legal requirements associated with these

- Users may download and print one copy of any publication from the public portal for the purpose of private study or research.

- You may not further distribute the material or use it for any profit-making activity or commercial gain

If the publication is distributed under the terms of Article $25 \mathrm{fa}$ of the Dutch Copyright Act, indicated by the "Taverne" license above, 
Rectal cancer

\title{
Residual metabolic tumor activity after chemo-radiotherapy is mainly located in initially high FDG uptake areas in rectal cancer
}

\author{
Jørgen van den Bogaard ${ }^{\mathrm{a}, 1}$, Marco H.M. Janssen ${ }^{\mathrm{a}, *, 1}$, G. Janssens ${ }^{\mathrm{b}}$, Jeroen Buijsen ${ }^{\mathrm{a}}$, Brigitte Reniers ${ }^{\mathrm{a}}$, \\ Philippe Lambin ${ }^{a}$, Guido Lammering ${ }^{\mathrm{a}, 1}$, Michel C. Öllers ${ }^{\mathrm{a}, 1}$ \\ a Department of Radiation Oncology (MAASTRO), GROW Research Institute, Maastricht, The Netherlands; ${ }^{\text {} C e n t e r ~ f o r ~ M o l e c u l a r ~ I m a g i n g ~ a n d ~ E x p e r i m e n t a l ~ R a d i o t h e r a p y ~(I M R E), ~}$ \\ Université Catholique de Louvain, Brussels, Belgium
}

\section{A R T I C L E I N F O}

Article history:

Received 22 December 2010

Received in revised form 6 April 2011

Accepted 8 April 2011

Available online 14 May 2011

\section{Keywords:}

Non-rigid image registration

Rectal cancer

FDG uptake

Residual disease

Morphons

\begin{abstract}
A B S T R A C T
Purpose: Recent literature suggests that tumor cells and areas within tumors with a high initial FDG uptake might be more resistant to (chemo)radiotherapy ((C)RT). This study was undertaken to test this hypothesis in rectal cancer using rigid and non-rigid image registration.

Patients and methods: Twenty-eight patients, diagnosed with locally advanced rectal cancer and referred for pre-operative treatment with CRT were included in this study. All patients underwent FDG-PET-CT imaging prior to and after CRT. Rigid and non-rigid image registration was performed to compensate organ deformations between the pre- and post-treatment PET-CT scans. The tumor was contoured on both PET-scans using SUV iso-contouring based on the SBR-method. The voxels with residual increased FDG uptake were studied and correlated to their pre-treatment FDG uptake level. Two SUV-volumehistograms were made based on the pre-treatment PET-data, one for the voxels within the pre-treatment tumor PET-based iso-contour and one for the voxels within the PET-based iso-contour of the residual tumor non-rigidly registered onto the pre-treatment scan.

Results: For the voxels with a pre-treatment FDG uptake of $>50 \%$ of SUV $_{\max }, 70.6 \pm 5.6 \%$ of the voxels were still metabolic active in the residual tumor, whereas for voxels with an FDG uptake of $<50 \%$ of $S U V_{\text {max }}$ only $51.1 \pm 6.7 \%$ were present in the metabolic active residual tumor.

Conclusion: This study presents areas in rectal tumors with an initially high FDG uptake to be most likely to show residual disease after CRT. This could indicate a higher (C)RT-resistance for tumor regions with a high FDG uptake prior to treatment.
\end{abstract}

(C) 2011 Elsevier Ireland Ltd. All rights reserved. Radiotherapy and Oncology 99 (2011) 137-141
For patients diagnosed with rectal cancer, pre-operative hypofractionated radiotherapy (RT) results in an improved local tumor control, whereas pre-operative treatment with chemo-radiotherapy (CRT) results in a significant downsizing and downstaging of the tumor [1-5]. In $15-30 \%$ of the patients being pre-operatively treated with CRT, even complete tumor regression is observed 68 weeks after completion of the CRT $[1-3,5,6]$. Also, patients with a pathological complete response (pCR) after pre-operative treatment with CRT have better long-term outcome than patients presenting with a pathological partial response (pPR) [7,8]. pCR might be indicative of a prognostically favorable biological tumor profile with a lower probability for local or distant recurrence and improved survival [7,8].

An important strategy to improve local tumor control might be escalating the radiation dose, in view of the fact that a higher

\footnotetext{
* Corresponding author. Address: MAASTRO Clinic, Dr. Tanslaan 12, 6201 BN Maastricht, Box 1588, The Netherlands.

E-mail address: marco.janssen@maastro.nl (M.H.M. Janssen).

1 These authors contributed equally.
}

radiation dose has been shown to yield a higher local control rate [9]. However, the increase of radiation dose is limited by the toxicity to surrounding normal tissues. One possibility to achieve a higher radiation dose to the tumor without additional damage to normal tissues would be to increase the dose to particular subparts of the tumor based on the intra-tumor heterogeneity visualized using functional imaging [10,11]. Molecular imaging studies already showed significant intra-tumor differences in perfusion, hypoxia, metabolism, cell density, and proliferation [12-17]. Different levels of radioresistance can exist in tumors $[10,11,18,19]$. Therefore, a non-uniform dose-distribution to the tumor seems logical. More therapy resistant areas within the tumor, regions suffering from hypoxia, low perfusion and/or high glucose metabolism or regions with a higher clonogenic cell density should receive a higher radiation dose while reducing the radiation dose to less radioresistant zones within the tumor and while keeping the normal tissue exposure equal. However, reducing the dose to less radioresistant zones within the tumor should be applied carefully to insure that the dose in these regions is not reduced to a 
dose-level below the clinically established dose to prevent from harmful undertreatment, which ultimately could result in a decreased probability of local tumor control. In pre-clinical research, an increase in the radiation dose showed a stronger increase of local control for tumors with higher FDG uptake compared to tumors with lower FDG uptake [20]. Therefore, we hypothesized that at intra-tumor level, high FDG uptake zones within the tumor at the pre-treatment performed PET-CT scan identify the location of the residual metabolic active areas after pre-operative CRT treatment. This hypothesis is by analogy in what Aerts et al. showed for nonsmall cell lung cancer (NSCLC) [21]. They proved that for NSCLC, residual metabolic activity was located within the high uptake zones on the initial FDG-PET-scan. If this hypothesis could be confirmed for rectal cancer, a higher rate of patients with complete tumor regression could possibly be expected when using an FDGuptake based redistribution of the radiation dose.

\section{Methods and materials}

\section{Patient characteristics}

For this study, twenty-eight patients diagnosed with nonmetastasized locally advanced rectal cancer (LARC) were included. All patients were pre-operatively treated with radiotherapy (28 fractions of $1.8 \mathrm{~Gy}, 5$ fractions/week) and concomitant chemotherapy (capecitabine $825 \mathrm{mg} / \mathrm{m}^{2}$ BID seven days/week), followed by a total mesorectal excision (TME) 7-8 weeks after the end of CRT. Radiotherapy treatment was delivered by four beams, AP, PA, and left and right lateral, each with energy of $10 \mathrm{MV}$. For each patient, a 3D conformal plan was made according to the ICRU 50 specifications $[22,23]$. As a part of the study, all patients underwent FDGPET-CT imaging both prior to and after pre-operative treatment, with the follow-up FDG-PET-CT scan performed 90 days after the onset of CRT, approximately 7-8 weeks after the last radiotherapy fraction. Since the main purpose of this study was to investigate the stability of high FDG uptake zones within rectum tumors during pre-operative CRT, patients who presented with a metabolic complete response (mCR) on the follow-up PET-CT scan were excluded from further analysis. According to Dutch law, the medical ethics committee approved the previous trial. All patients gave written informed consent before they were entered into the study.

\section{PET-CT imaging and processing}

After a fasting period of at least $6 \mathrm{~h}$, FDG was injected intravenously, with the activity normalized for the weight of the patient (weight $[\mathrm{kg}] * 4+20[\mathrm{MBq}]$ ). After an uptake period of $60 \mathrm{~min}$, PET acquisition was started. Patients were positioned in supine position on the flat carbon table top equal to the radiotherapy treatment position. Both legs were supported and fixed by a combifix. Reference points were located with the help of a movable laser alignment system (LAP GmbH, Lüneburg, Germany) and reference lines were set down on the patient. All PET-CT scans were performed using a dedicated Siemens Biograph 40 TruePoint PET-CT simulator (Siemens Medical, Erlangen, Germany) with an axial field of view of $16.2 \mathrm{~cm}$, a reconstructed slice thickness of $3 \mathrm{~mm}$ and a pixel spacing of $5.3456 \mathrm{~mm}$ in both directions. The scanner is equipped with ultra-fast detector electronics (Pico3D) and has a spatial resolution of approximately $4.5 \mathrm{~mm}$ full-width-at-halfmaximum (FWHM). PET-imaging was performed in 3D, requiring a proper scatter correction. CT-based attenuation and decay correction was performed. PET images were reconstructed from the acquired list-mode (LM) data using Fourier-rebinning (FORE) and 2D ordered-subset-expectation-maximization-reconstruction (OSEM 2D) with four iterations and eight subsets.

\section{Image registration}

3D image registration was used to compensate for the motion between the follow-up PET-CT scan performed after treatment and the PET-CT scan performed prior to treatment. First, an automatic rigid image registration was performed to align the two PETCT scans, based on minimization of the sum of squared intensity differences of the two CT-scans. Then, a CT-based non-rigid registration algorithm was used in order to estimate local deformations between the rigidly aligned PET-CT-scans. These deformations were estimated using a multi-scale non-parametric registration method called Morphons [24-26], by optimizing the matching between local structures in both CT-images, along with a deformation regularity criterion. A total of eight scales were used, each with a maximum of 20 optimization iterations. To visualize the performance of both rigid and non-rigid registrations, difference images were calculated by subtraction of the images intensities of corresponding voxels within the two images. Difference images were inspected for three situations: pre-treatment and follow up CT-images, rigid registered pre-treatment and follow-up CTimages and rigid and non-rigidly registered pre-treatment and follow-up CT-images. The deformation field resulting from rigid and non-rigid registrations was then applied on the follow-up PET-scan to study the stability of high FDG uptake zones within rectal tumors during pre-operative treatment with CRT.

\section{Image analysis}

To study the stability of areas with high FDG uptake within the tumor, the tumor was automatically delineated on the pre-treatment and post-treatment PET-scan, based on standardized-uptake-value (SUV) thresholding. For each PET-scan, the tumor-tobackground signal ratio was determined from which the optimal contouring threshold (percentage of the maximal SUV in the tumor) was determined. Details on this method have been described before $[27,28]$. Within the PET-based tumor contour, the voxels were arranged into a histogram of 10 bins based on the pre-treatment FDG uptake level of the tumor cells within the voxel. Voxels with relative low FDG uptake levels were assigned to SUVbin 1 and the voxels with the highest FDG uptake levels were assigned to SUVbin 10. The SUV-volume-histograms give an insight into the intra-tumor heterogeneity of the FDG uptake. Two 10 bin SUVvolume-histograms were made based on the pre-treatment PETscan, one for the voxels within the pre-treatment tumor iso-contour and one for the voxels within the deformed post-treatment iso-contour of the residual tumor projected onto the pre-treatment PET-scan. The decrease of the amount of voxels per SUV-bin was studied to test the hypothesis that tumor cells presenting with a high FDG uptake are less sensitive to (chemo)radiotherapy. Confirmation of this hypothesis would lead to a high decrease of the amount of voxels within the bins of the histograms representing the low SUV-regions (SUVbin 1-3), whereas the amount of voxels within the high SUV-regions (SUVbin 7-10) would remain stable.

\section{Results}

Twenty-eight patients (mean age: $66 \pm 11$ (range: 42-89) years, 17 males, 11 females), diagnosed with LARC, were included in this study. Four of the included patients presented with a $\mathrm{MCR}$ on the follow-up PET-CT scan performed prior to surgery, excluding them from further analysis.

Non-rigid registration and analysis of the sequential FDG-PET$\mathrm{CT}$ images were performed for the 24 patients presenting with a metabolic partial response (mPR). From the pre-treatment FDGPET scan, an average PET-positive tumor volume of $43.2 \pm$ $4.9 \mathrm{~cm}^{3}$ (range: $8.6-91.5 \mathrm{~cm}^{3}$ ) was observed, whereas the average 
PET-positive residual volume was $22.2 \pm 3.2 \mathrm{~cm}^{3}$ (range: $4.4-$ $54.9 \mathrm{~cm}^{3}$ ). From the pre-treatment FDG-PET-CT scan, an average $S \mathrm{VV}_{\max }$ of $14.4 \pm 1.0$ (range: 6.9-22.7) was found, whereas from the follow-up FDG-PET-CT scan an average SUV $_{\max }$ of $6.1 \pm 0.4$ (range: 3.5-10.1) was found.

In Fig. 1, the pre-treatment PET-CT scan of a patient is shown in the top left figure (Fig. 1A), whereas the rigid registered and nonrigid registered follow-up PET-CT scans are shown in, respectively, the top right (Fig. 1B) and bottom left figure (Fig. 1C), with in green the tumor contours resulting from SUV-thresholding. Note the difference in rectal filling between the pre-treatment and rigid registered follow-up PET-CT scan. When using only rigid image registration, a reliable region-based or voxel-wise comparison of the two PET-CT scans was not possible. However, by applying the morphons based non-rigid registration algorithm, the difference in rectal filling between the two PET-CT scans was corrected for, enabling a voxel-wise comparison of the two PET-CT scans. The bottom right part of Fig. 1 (Fig. 1D) displays the non-rigid registered PET-based tumor contour (in blue) of the residual disease projected on the pre-treatment PET-CT scan to study the pre-treatment FDG uptake levels of the voxels of the residual disease.

For each of the included patients, two SUV-volume-histograms, each of 10 bins, were made, one for the voxels within the pretreatment tumor iso-contour and one for the voxels within the deformed follow-up tumor contour projected onto the pre-treatment PET-data. The voxels within each tumor contour were assigned to one of the 10 bins (low to high FDG uptake from SUVbin 1-10), based on their FDG uptake level on the FDG-PET-data acquired prior to treatment. The average SUV-volume-histograms for both situations are displayed in Fig. 2A. Note the large decrease of the amount of voxels within the low-SUV bins on the left site of the histogram (SUVbin 1-3) compared to the stable amounts of voxels present within the high-SUV bins (SUVbin 7-10) from the pretreatment to the post-treatment situation. This indicates that the metabolic active residual tumor mainly consists of tumor cells with relatively high pre-treatment FDG uptake levels, indicating an increased radioresistance of cells with a high pre-treatment FDG uptake (Fig. 2B). For the bins of the histograms containing the voxels within a pre-treatment FDG uptake of $>50 \%$ of $S U V_{\max }$ (Fig. 2B, light gray boxes), $70.6 \pm 5.6 \%$ of the voxels were still met- abolic active in the residual tumor, whereas for the bins of the voxels with an FDG uptake of $<50 \%$ of $\operatorname{SUV}_{\max }$ (Fig. 2B, dark gray boxes), $51.1 \pm 6.7 \%$ of the voxels were present in the metabolic active residual tumor.

\section{Discussion}

In radiation oncology, there is a growing interest in non-uniform dose distribution techniques like dose painting or sub-volume boosting to increase the rate of local tumor control [10$13,15,29]$. In non-small cell lung cancer, promising results have been presented showing that individualized dose prescription based on normal tissue constraints is safe while improving survival rates compared to traditional (chemo)radiotherapy schemes [9]. Selective irradiation of tumor sub-volumes of potentially radioresistant regions within the tumor along with a decrease in radiation dose to less resistant regions could even increase local tumor control rates with equal or decreased levels of side-effects. However, before the potential of non-uniform dose distributions can be used in clinical practice for rectal cancer patients, the regions in the tumor with an increased resistance to treatment should be defined accurately. The results of this study indicate that residual disease is mainly located in the area with highest FDG uptake on the pre-treatment PET scan, suggesting that these areas are more resistant to the anti-cancer treatment. However, due to organ deformations between the pre-treatment and follow-up PET-CT scans, like differences in bladder and bowel filling, the use of rigid image registration alone was not sufficient to align the two PET-CT scans. To account for organ deformations between the pre- and post-treatment CT scan, a morphons based non-rigid image registration algorithm was used. Although we believe that the non-rigid image registration method used in this study is able to accurately estimate organ motion in the abdomen even in cases of large anatomical deformations between pre- and post-therapy scans, a thorough validation becomes difficult when dealing with treatment related tumor shrinkage. This is a general and difficult problem with all non-rigid registration algorithms that would need to be addressed in the future. Also, in order to monitor treatment related tumor responses, like tumor shrinkage, during therapy, repeated FDG PET-CT imaging and daily pre-treatment verification using
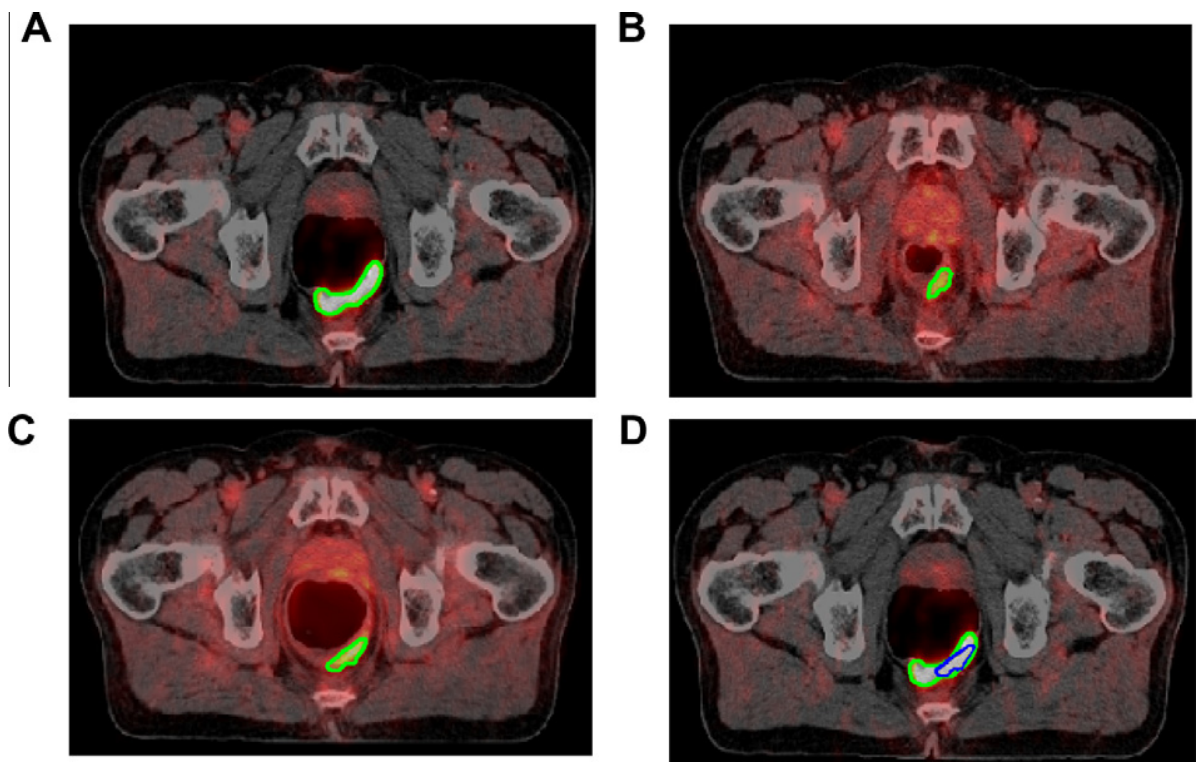

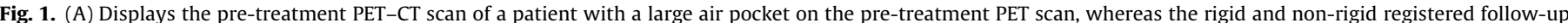

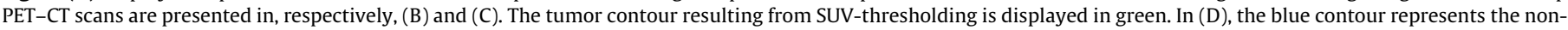
rigid registered PET-based contour of the residual tumor jprojected on the pre-treatment PET-CT scan. 

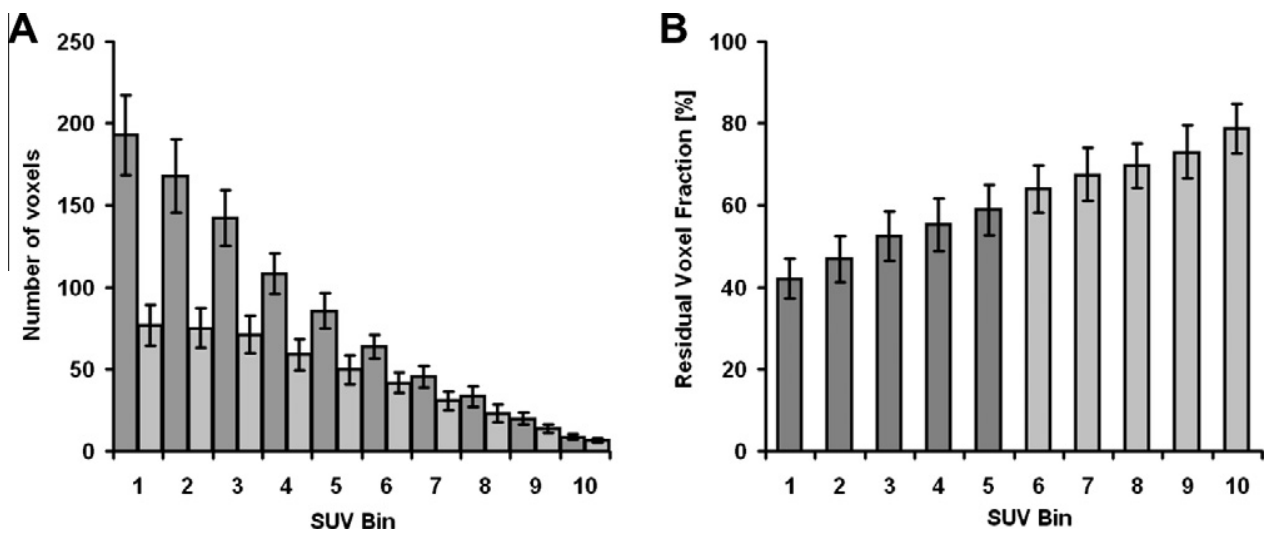

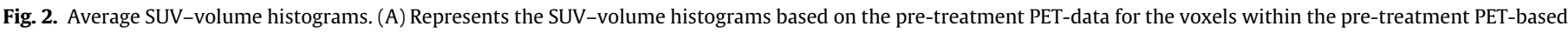

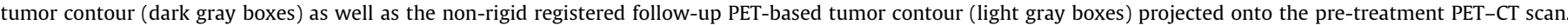

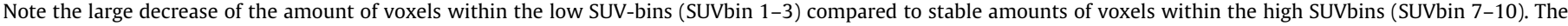

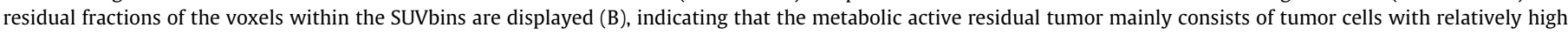
FDG uptake levels prior to treatment.

$\mathrm{kV} / \mathrm{MV}$ imaging on the linac are necessary to adapt treatment plans when necessary. Especially for sub-volume boosting with radiotherapy, accurate knowledge about the orientation and location of the tumor is required prior to each radiotherapy fraction.

In this study we found that residual disease mainly occurs at the locations within the tumor presenting with high FDG uptake on the pre-treatment FDG-PET scan. Recently, an interesting relationship has been presented between FDG uptake in lung tumors and the probability of tumor control tumor 3 months after the start of radiotherapy [30]. They showed that the voxel control probability (VCP) decreased for increasing FDG uptake indicating that areas with high FDG uptake are more resistant to current radiotherapy schemes. A relationship between the occurrence of residual disease after therapy and the total amount of radiation dose seems obvious, but such a relationship was not found in this study.

For rectal cancer, pCR after pre-operative treatment with CRT was found to result in better long-term outcome, a low probability for local and distant recurrence as well as improved survival when compared to the patients presenting with a partial response $[7,8]$. However, to our knowledge, the clinical relevance of residual metabolic (PET-positive) uptake after treatment with CRT was not yet demonstrated. Relationships between residual metabolic uptake and long-term outcome and the probability for recurrence have not yet been proven for rectal cancer. Therefore, a (multi-centric) study on response prediction for rectal cancer is started using multi-variate analysis with nomograms [31]. Preliminary results show that one of the criteria found to be highly predictive for the probability on distant metastases and local recurrence within 5 years was the presence of residual (PET-positive) disease after pre-operative treatment with CRT.

One of the limitations of FDG-PET based response monitoring is the increased FDG uptake in (peritumoral) inflammatory cells induced by radiotherapy treatment [5]. Based on only static FDGPET-data it is nearly impossible to differentiate between tumor and inflammation. However, the presence of inflammatory responses can be visually observed as a large PET positive volume with a high, but more diffuse FDG uptake [5,32,33]. In an earlier published study on PET-based response monitoring the influences of inflammatory responses on the quantitation of the metabolic treatment response for rectal tumors during and after pre-operative treatment with CRT was presented [34]. Most of the inflammatory responses were visually observed from the FDG-PET images acquired two weeks after the onset of pre-operative treatment with CRT, whereas at the FDG-PET images acquired 90 days after the onset of treatment, approximately 7-8 weeks after the last radiotherapy fraction, less inflammatory tissue was observed for these patients. For the patients with a visually observed peritumoral inflammatory response on the PET-images, the inflammatory response was also reported by the pathologist after examination of the resected specimen. For none of the patients included in this study an inflammatory response was visually observed from the PET-images. Also, no inflammatory responses were reported by the pathologist after examination of the resected specimen.

In conclusion, the presented results show that high FDG uptake regions within rectal tumors prior to chemo-radiotherapy can identify the location of the residual metabolic active areas after treatment. If these zones could be located with high accuracy, dose escalation in high FDG uptake zones within the tumor is expected to result in a higher rate of local tumor control or even a complete pathological tumor regression after pre-operative treatment with chemo-radiotherapy.

\section{Conflicts of interest statements}

We are not aware of any actual or potential conflicts of interest.

\section{References}

[1] Valentini V et al. Ten years of preoperative chemoradiation for extraperitoneal T3 rectal cancer: acute toxicity, tumor response, and sphincter preservation in three consecutive studies. Int J Radiat Oncol Biol Phys 2001;51:371-83.

[2] Capirci $C$ et al. Sequential FDG-PET/CT reliably predicts response of locally advanced rectal cancer to neo-adjuvant chemo-radiation therapy. Eur J Nucl Med Mol Imaging 2007;34:1583-93.

[3] Capirci $C$ et al. Long-term prognostic value of 18F-FDG PET in patients with locally advanced rectal cancer previously treated with neoadjuvant radiochemotherapy. AJR Am J Roentgenol 2006;187:W202-8.

[4] Janssen $\mathrm{MH}$ et al. Evaluation of early metabolic responses in rectal cancer during combined radiochemotherapy or radiotherapy alone: sequential FDGPET-CT findings. Radiother Oncol 2010;94:151-5.

[5] Janssen $\mathrm{MH}$ et al. Accurate prediction of pathological rectal tumor response after two weeks of preoperative radiochemotherapy using (18)Ffluorodeoxyglucose-positron emission tomography-computed tomography imaging. Int J Radiat Oncol Biol Phys 2009;77:392-9.

[6] Vliegen RF et al. Can an FDG-PET/CT predict tumor clearance of the mesorectal fascia after preoperative chemoradiation of locally advanced rectal cancer? Strahlenther Onkol 2008;184:457-64.

[7] Eich HT et al. Neoadjuvant radiochemotherapy and surgery for advanced rectal cancer: prognostic significance of tumor regression. Strahlenther Onkol 2011:187:225-30.

[8] Maas $\mathrm{M}$ et al. Long-term outcome in patients with a pathological complete response after chemoradiation for rectal cancer: a pooled analysis of individual patient data. Lancet Oncol 2010;11:835-44.

[9] van Baardwijk A et al. Mature results of an individualized radiation dose prescription study based on normal tissue constraints in stages I to III nonsmall-cell lung cancer. J Clin Oncol 2010;28:1380-6. 
[10] Petit SF et al. Metabolic control probability in tumour subvolumes or how to guide tumour dose redistribution in non-small cell lung cancer (NSCLC): an exploratory clinical study. Radiother Oncol 2009;91:393-8.

[11] Petit SF, et al. [(18)F]fluorodeoxyglucose uptake patterns in lung before radiotherapy identify areas more susceptible to radiation-induced lung toxicity in non-small-cell lung cancer patients. Int J Radiat Oncol Biol Phys, 2010; doi:10.1016/j.ijrobp.2010.06.016.

[12] Bentzen SM. Dose painting and theragnostic imaging: towards the prescription, planning and delivery of biologically targeted dose distributions in external beam radiation oncology. Cancer Treat Res 2008;139:41-62.

[13] Bentzen SM. Theragnostic imaging for radiation oncology: dose-painting by numbers. Lancet Oncol 2005;6:112-7.

[14] Vanderstraeten B et al. [18F]fluoro-deoxy-glucose positron emission tomography ([18F]FDG-PET) voxel intensity-based intensity-modulated radiation therapy (IMRT) for head and neck cancer. Radiother Oncol 2006;79:249-58.

[15] Tanderup K, Olsen DR, Grau C. Dose painting: art or science? Radiother Oncol 2006;79:245-8.

[16] Piert $\mathrm{M}$ et al. Hypoxia-specific tumor imaging with 18F-fluoroazomycin arabinoside. J Nucl Med 2005;46:106-13.

[17] Foo SS et al. Functional imaging of intratumoral hypoxia. Mol Imaging Biol 2004;6:291-305.

[18] Velazquez ER et al. Prediction of residual metabolic activity after treatment in NSCLC patients. Acta Oncol 2010;49:1033-9.

[19] Aerts $\mathrm{HJ}$ et al. Stability of $18 \mathrm{~F}$-deoxyglucose uptake locations within tumor during radiotherapy for NSCLC: a prospective study. Int J Radiat Oncol Biol Phys 2008;71:1402-7.

[20] Schutze $C$ et al. Effect of increase of radiation dose on local control relates to pre-treatment FDG uptake in FaDu tumours in nude mice. Radiother Oncol 2007;83:311-5.

[21] Aerts $\mathrm{HJ}$ et al. Identification of residual metabolic-active areas within individual NSCLC tumours using a pre-radiotherapy (18)FluorodeoxyglucosePET-CT scan. Radiother Oncol 2009;91:386-92.
[22] ICRU report 50. Prescribing, Recording, and Reporting Photon Beam Therapy. 1993.

[23] ICRU report 50. Prescribing, Recording, and Reporting Photon Beam Therapy (Supplement to ICRU 50). ICRU report 62. 1999.

[24] Knutsson H, Andersson M, Morphons: segmentation using elastic canvas and paint on priors. Proc IEEE Int Conf Image Process 2005:1226-9.

[25] Janssens, G. Diffeomorphic registration of images with variable contrast enhancement. Int J Biomed Imaging, 2011:2011.

[26] Janssens G. Evaluation of nonrigid registration models for interfraction dose accumulation in radiotherapy. Med Phys 2009;36:4268-76.

[27] Daisne JF et al. Tri-dimensional automatic segmentation of PET volumes based on measured source-to-background ratios: influence of reconstruction algorithms. Radiother Oncol 2003;69:247-50.

[28] Ollers $M$ et al. The integration of PET-CT scans from different hospitals into radiotherapy treatment planning. Radiother Oncol 2008;87:142-6.

[29] Lambin P et al. The ESTRO Breur lecture 2009. From population to voxel-based radiotherapy: exploiting intra-tumour and intra-organ heterogeneity for advanced treatment of non-small cell lung cancer. Radiother Oncol 2010;96:145-52.

[30] Petit SF et al. Intra-voxel heterogeneity influences the dose prescription for dose-painting with radiotherapy: a modelling study. Phys Med Biol 2009;54:2179-96.

[31] van Stiphout RG et al. Development and external validation of a predictive model for pathological complete response of rectal cancer patients including sequential PET-CT imaging. Radiother Oncol 2011;98:126-33.

[32] Larson SM. Cancer or inflammation? A Holy Grail for nuclear medicine. J Nucl Med 1994;35:1653-5.

[33] Kao PF, Chou YH, Lai CW. Diffuse FDG uptake in acute prostatitis. Clin Nucl Med 2008;33:308-10.

[34] Janssen $\mathrm{MH}$ et al. Accurate prediction of pathological rectal tumor response after two weeks of preoperative radiochemotherapy using (18)Ffluorodeoxyglucose-positron emission tomography-computed tomography imaging. Int J Radiat Oncol Biol Phys 2010;77:392-9. 